\title{
Two Cases of Bovine Sarcoma in Clinically Long-Standing Lesions
}

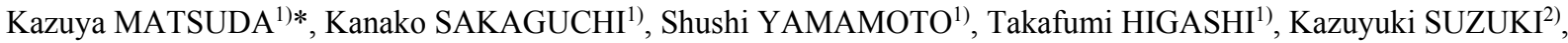 \\ Kiyoshi TAGUCHI ${ }^{2)}$, Masateru KOIWA ${ }^{2)}$ and Hiroyuki TANIYAMA ${ }^{1)}$

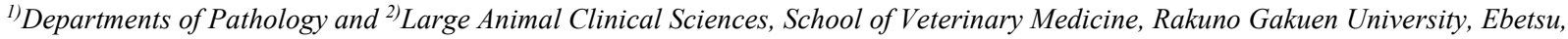 \\ Hokkaido 069-8501, Japan
}

(Received 23 April 2008/Accepted 14 October 2008)

ABSTRACT. Histopathological examination of clinically long-standing lesions with durations of one year or more in the extremities of two cattle revealed the presence of sarcomas with distant metastases. In case 1, neoplastic cells were fusiform to pleomorphic, stained for no specific differentiation markers, and diagnosed as undifferentiated sarcoma. Neoplastic growth in case 2 was composed of spindle to histiocytoid cells and a significant number of multinucleated giant cells, both of which were immunoreactive to histiocyte markers, and diagnosed as giant cell malignant fibrous histiocytoma. Neoplastic cells of both cases were immunohistochemically positive for nitric oxide-related antigens, which were recognized as markers of inflammation-induced carcinogenesis in human and laboratory animals.

KEY WORDS: bovine, inflammation, neoplasm, nitric oxide.

In cattle, swellings or masses formed in the extremities, especially around the joints, are usually recognized as inflammatory reactions because of the frequency of arthritis and periarthritis as a result of infection and the rarity of tumors in this species. In this report, we present two cases of sarcoma found in clinically long-standing lesions on the extremities, and discuss the possible association between chronic inflammation and carcinogenesis, besed on immunohistochemical detection of nitric oxide (NO)-related antigens.

Case 1 was a 7-year-old female Holstein-Friesian cow. This animal presented with swelling of outside of the left tarsal joint from one year previously, and the lesion had gradually enlarged. In the necropsy, the mass was about 30 $\mathrm{cm}$ in diameter. On the cut surface, the mass had an extensive area of hemorrhage and degeneration in the center, compartmentalized with fibrous connective tissue (Fig. 1A). There were several brownish-yellow nodules outside the central degenerative tissue. The left medial iliac lymph nodes were moderately enlarged.

Histopathologically, the nodules were composed of neoplastic growth. The majority of neoplastic cells were fusiform with eosinophilic cytoplasm without distinct cell boundaries, and ovoid to elongated nucleus with prominent nucleoli, and were arranged in a fascicular fashion (Fig. 1B). Partially, neoplastic growth of cells with atypical nuclei and pleomorphic eosinophilic, broad cytoplasm was present (Fig. 1C). Multinucleated tumor cells with central chains of nuclei were rarely observed. Mitoses were frequent. There was diffuse mild infiltration of lymphocytes and lymphoid follicles in and around the neoplastic tissue, respectively. The left medial iliac lymph node was largely replaced with

\footnotetext{
* Correspondence to: Matsuda, K., Department of Pathology, School of Veterinary Medicine, Rakuno Gakuen University, Bunkyodai-Midorimachi 582, Ebetsu 069-8501, Japan. e-mail:kmatsuda@rakuno.ac.jp
}

pleomorphic neoplastic cells similar to those in the tarsal mass (Fig. 1D). Immunohistochemical staining was performed in paraffin-embedded sections by an avidin-biotin complex procedure. The primary antibodies used in this study are listed in Table 1. Neoplastic cells were positive for vimentin, desmin and nestin, but negative for $\alpha$-smooth muscle actin (SMA), myoglobin, myosin and S-100 protein (Table 2). Rhabdomyosarcoma was preferred as differential diagnosis for immunoreactivity to desmin and presence of tumor cells with nuclear chains; however, this case was diagnosed as undifferentiated sarcoma based on lack of myoglobin staining.

To detect NO-related antigen expression, we performed immunohistochemistry on cryostat sections with antibodies against inducible nitric oxide synthase (iNOS), nitroguanine (NG) and nitrotyrosine (NT). In addition, we investigated cyclooxygenase 2 (COX2) and p53 expression on paraffinembedded sections. The neoplastic cells were diffusely positive for iNOS and NT, and infiltrating neutrophils were also stained (Table 2). A positive reaction to $\mathrm{NG}$ was detected in the cytoplasm but less in the nuclei of some neoplastic cells, especially in the mitotic phase. Neoplastic cells were also stained for COX2 and p53 in the cytoplasm and nuclei, respectively.

Case 2 was a 6-year-old female Holstein-Friesian cow with swelling of the anterior surface of the right fetlock joint noticed 15 months previously. From three months before necropsy, granulation-like soft tissue had massively prolifereated in the lesion. In the necropsy, the right fetlock joint was swollen to about $15 \mathrm{~cm}$ in diameter, and pale yellow tissue was observed on the anterior surface (Fig. 2A). On the cut surface, several pale yellow to pale brown solid masses were located outside of the metacarpophalangeal joint. The frontal surface of the right metacarpal bone showed extraperiosteal bone formation. There were several white nodules, up to $3 \mathrm{~cm}$ in diameter, in the right cervical 

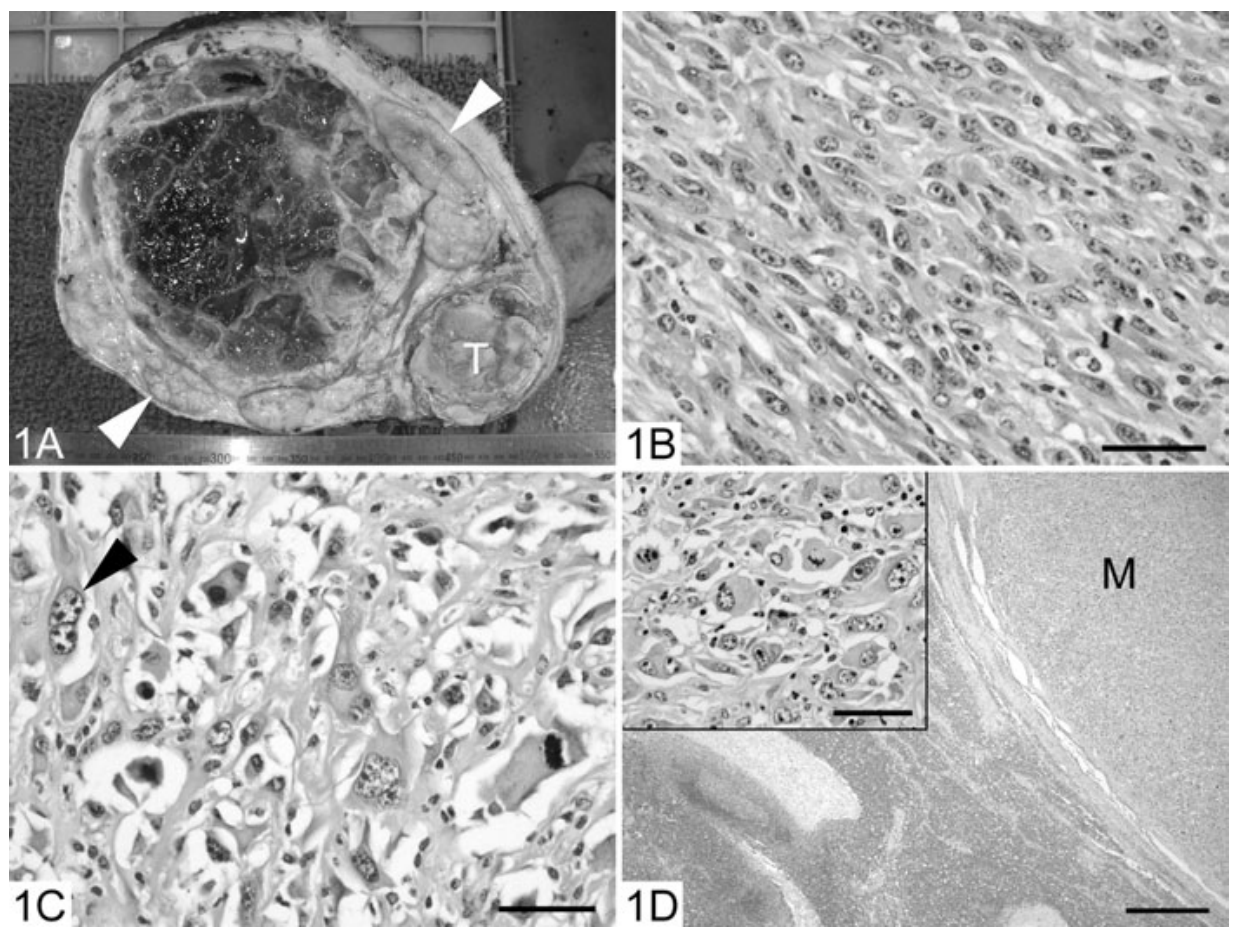

Fig. 1. Undifferentiated sarcoma in case 1. (A) Transverse cut surface at left tarsal joint (T). Neoplastic masses (arrowheads) located outside the central degenerative tissue. (B) Tarsal mass. Fusiform tumor cells without distinct cell boundaries are arranged unidirectionally. Hematoxylin and eosin (HE) stain. Bar=50 $\mu \mathrm{m}$. (C) Tarsal mass. Pleomorphic tumor cells showing atypical nuclear morphology, rarely arranged in chains (arrowhead), and relatively-broad cytoplasm. HE stain. Bar $=50 \mu \mathrm{m}$. (D) Left medial iliac lymph node. Metastatic growth $(\mathrm{M})$ of undifferentiated sarcoma in the lymph node. HE stain. Bar $=500 \mu \mathrm{m}$. (Inset) Higher magnification of metastatic undifferentiated sarcoma. Pleomorphic tumor cells with atypical nucleus/nuclei and broad cytoplasm, similar to cells shown in Fig. 1C. HE stain. Bar $=50 \mu \mathrm{m}$.

Table 1. Primary antibodies used for immunohistochemical examinations

\begin{tabular}{|c|c|c|c|c|}
\hline Marker & Isotype (clone) & Dilution & Antigenic retrieval & Manufacture \\
\hline Vimentin & $\mathrm{mAb}(\mathrm{V} 9)$ & 1 in 100 & $\mathrm{MW}^{\mathrm{a})}$ & Progen, Heidelberg, Germany \\
\hline Desmin & $\mathrm{mAb}(\mathrm{D} 9)$ & 1 in 40 & MW & Progen, Heidelberg, Germany \\
\hline Nestin & $\mathrm{pAb}$ & 1 in 50 & None & Immuno-Biological Laboratories, Gunma, Japan \\
\hline SMA & $\mathrm{mAb}(1 \mathrm{~A} 4)$ & 1 in 10000 & None & DakoCytomation, Glostrup, Denmark \\
\hline Myoglobin & $\mathrm{pAb}$ & 1 in 200 & Pepsin' ${ }^{\text {b) }}$ & DakoCytomation, Glostrup, Denmark \\
\hline Myosin & $\mathrm{mAb}(\mathrm{HSM}-\mathrm{V})$ & 1 in 500 & Pepsin & Sigma, Saint Louis, MO, U.S.A. \\
\hline S-100 & $\mathrm{pAb}$ & 1 in 1000 & MW & DakoCytomation, Glostrup, Denmark \\
\hline Lysozyme & $\mathrm{pAb}$ & 1 in 100 & None & Lab Vision, Fremont, CA, U.S.A. \\
\hline CD68 & $\mathrm{mAb}(\mathrm{ED} 1)$ & 1 in 100 & MW & Serotec, Oxford, UK \\
\hline CD3 & $\mathrm{pAb}$ & Prediluted & $\mathrm{AC}^{\mathrm{c})}$ & DakoCytomation, Glostrup, Denmark \\
\hline iNOS & $\mathrm{pAb}$ & 1 in 50 & MW & BD Biosciences, San Jose, CA, U.S.A. \\
\hline NG & $\mathrm{pAb}$ & 1 in 50 & MW & Dojindo Laboratories, Kumamoto, Japan \\
\hline NT & $\mathrm{pAb}$ & 1 in 50 & MW & Upstate, Lake Placid, NY, U.S.A. \\
\hline $\mathrm{COX} 2$ & $\mathrm{pAb}$ & 1 in 1500 & MW & Cayman Chemical, Ann Arbor, MI, U.S.A. \\
\hline p53 & $\mathrm{pAb}$ & 1 in 200 & MW & Signet Laboratories, Dedham, MA, U.S.A. \\
\hline
\end{tabular}

a) Microwave in $0.01 \mathrm{M}$ citrate buffer, $\mathrm{pH} 6.0,500 \mathrm{~W}, 15 \mathrm{~min}$. b) $0.4 \%$ pepsin in $0.01 \mathrm{M} \mathrm{PBS}, 37^{\circ} \mathrm{C}, 30 \mathrm{~min}$. c) Autocrave in $0.01 \mathrm{M}$ citrate buffer, $\mathrm{pH} 6.0,121^{\circ} \mathrm{C}, 15 \mathrm{~min}$.

SMA, $\alpha$-smooth muscle actin, iNOS, inducible nitric oxide synthase, NG, nitroguanine, NT, nitrotyrosine, COX2, cyclooxygenase $2, \mathrm{mAb}$, mouse monoclonal antibody, pAb, rabbit polyclonal antibody.

lymph nodes and lungs.

The metacarpophalangeal masses and the nodules in the lymph nodes and lungs were histopathologically similar.
These tissues were composed of neoplastic proliferation of spindle to histiocytoid cells admixed with a large population of multinucleated giant cells. The spindle tumor cells were 
Table 2. Results of immunohistochemical staining in two bovine sarcomas

\begin{tabular}{lcc}
\hline Antigen & Case 1 & Case 2 \\
\hline Vimentin & +++ & $+++/+++*$ \\
Desmin & +++ & $-/-$ \\
Nestin & ++ & $-/-$ \\
SMA & - & $-/-$ \\
Myoglobin & - & ND \\
Myosin & - & ND \\
S-100 & - & $-/-$ \\
Lysozyme & ND & $++/+++$ \\
CD68 & ND & $+/+++$ \\
iNOS & ++ & $+++/+++$ \\
NG & + & $++/++$ \\
NT & +++ & $+++/+++$ \\
COX2 & ++ & $++/+$ \\
p53 & ++ & $+/+$ \\
\hline
\end{tabular}

+++ , positive in $>70 \%$ tumor cells, ++ , positive in $30-70 \%$ tumor cells, + , positive in $<30 \%$ tumor cells, - , negative, ND, not done.

* Results in mononuclear/multinucleated tumor cells. SMA, $\alpha$-smooth muscle actin, iNOS, inducible nitric oxide synthase, NG, nitroguanine, NT, nitrotyrosine, COX2, cyclooxygenase 2 . loosely arranged in a streaming fashion with collagenous stroma (Fig. 2B). The histiocytoid cells had abundant eosinophilic cytoplasm and an eccentric nucleus, and sometimes had a large vesicular nucleus with prominent nucleolus (Figs. 2C and 2D). The giant cells were quite pleomorphic and had many nuclei, up to 60 in a $4 \mu \mathrm{m}$ section. Mitotic figures were frequently observed in both mononuclear and multinucleated tumor cells. Multifocal necrosis and cavitation were observed. There was diffuse mild to moderate lymphocyte infiltration within the neoplastic tissue. Immunohistochemically, both mononuclear and multinucleated neoplastic cells were positive for vimentin, lysozyme and CD68 but negative for desmin, nestin, SMA and S-100 protein. Alkaline phosphatase reaction performed on cryostat sections revealed the absence of reactivity. From these results, this case was diagnosed as malignant fibrous histiocytoma of giant cell type, also called as giant cell tumor of soft parts [2]. The mononuclear and multinucleated neoplastic cells were stained for all NO-related antigens investigated, and for COX2 and p53 in the cytoplasm and nuclei, respectively (Table 2 ).

Histopathlogical investigations revealed the presence of sarcomas in the lesions in the limbs and distant metastases in
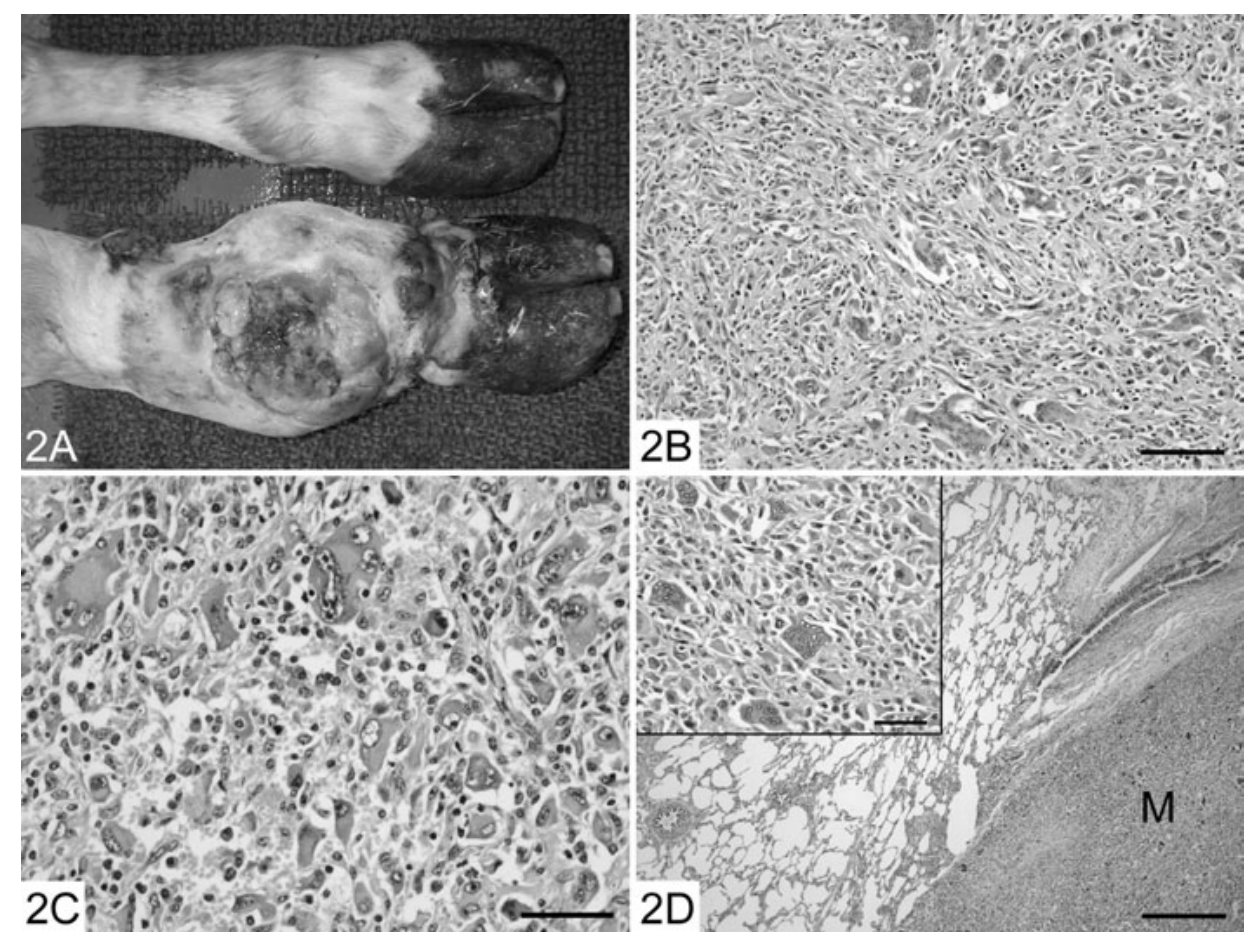

Fig. 2. Giant cell malignant fibrous histiocytoma (MFH) in case 2. (A) Swelling of the right fetlock joint due to neoplasia. (B) Metacarpophalangeal mass. Proliferation of spindle tumor cells in a looselystreaming fashion admixed with multinucleated giant cells. Diffuse mild infiltration of lymphocytes is present. HE stain. Bar $=100 \mu \mathrm{m}$. (C) Metacarpophalangeal mass. Histiocytoid tumor cells with eccentric nucleus and giant cells with atypical nuclei. Diffuse moderate infiltration of lymphocytes are present. HE stain. Bar=50 $\mu \mathrm{m}$. D) Lung. Metastatic growth (M) of giant cell MFH. HE stain. Bar=500 $\mu \mathrm{m}$. (Inset) Higher magnification of metastatic giant cell MFH. Proliferation of fusiform to histiocytoid neoplastic cells and multinucleated giant cells. HE stain. Bar $=50 \mu \mathrm{m}$. 
two cows. From the size and distribution of the neoplastic growth, it was considered that the extremities were the primary neoplastic foci in both cases. Sarcomas are rare conditions in bovine species [2]. The tumors were formed in the lesions with long clinical course of one year or more, and the lesions adjacent to the joints in the extremities were subjected to exogenous physical stimuli and infection. Hence, the relationship of persistent inflammation and stimulus to carcinogenesis and/or tumor progression was suspected.

Chronic inflammation has been recognized as an important factor in carcinogenesis, such as gastric adenocarcinoma in Helicobacter pylori-induced gastritis, hepatocellular carcinoma in hepatitis virus infections, squamous cell carcinoma in oral lichen planus in humans, and in animal models such as cholangiocarcinoma in liver fluke infection and colon cancer in inflammatory bowel diseases $[1,3]$. In these instances, $\mathrm{NO}$ and relative reactive nitrogen and oxygen species are capable of tumor initiation and/or progression by nitrative and oxidative damage e.g. DNA damage, inhibition of DNA repair enzymes, functional activation of oncogene products, and functional inactivation of tumor-suppressor gene products $[1,6]$. Significant amount of NO is generated via iNOS, so iNOS is identified as a biomarker for local excess production of NO. Additionally, NG and NT are used as markers of nitrative DNA and protein damage by $\mathrm{NO}$, respectively $[3,6]$. COX2 is induced by $\mathrm{NO}$ and causes neovascularization via prostaglandin upregulation and promotes tumor progression [5]. Excess NO can accumulate p53 which lack the binding capacity to DNA through the modification of p53 by NO [6]. Mutations of p53 are positively correlated with excess NO in inflammatory bowel diseases [4]. In the present cases, immunohistochemistry revealed NO generation by neoplastic cells and consequent nitrative DNA and protein damage. Moreover, the cells were also positive for COX2 and p53. These findings might suggest the possible association between persistent traumatic stimuli, inflammation, tissue repair, and neoplastic transformation and/or tumor progression in the present cases, although there have been no reports on the significance of immunohistochemical detection of NOrelated antigens in bovine neoplasms.

In large animals, swellings on the skin, especially the extremities, are subjected to external physical irritation and subsequent infection, and animal weight and movement can be a stimulus, depending on the affected site, which creates a vicious cycle of persistent local inflammation and increases the mass size, causing more susceptibility to stimuli. In many bovine cases, swelling or masses on the skin tend to be recognized as inflammatory reactions and are rarely served to histopathological examination; however, persistence of these lesions may be a predisposing factor to neoplastic conversion and progression.

\section{REFERENCES}

1. Chaiyarit, P., Ma, N., Hiraku, Y., Pinlaor, S., Yongvanit, P., Jintakanon, D., Murata, M., Oikawa, S. and Kawanishi, S. 2005. Nitrative and oxidative DNA damage in oral lichen planus in relation to human oral carcinogenesis. Cancer Sci. 96: 553-559.

2. Goldschmidt, M. H. and Hendrick, M. J. 2002. Tumors of the skin and soft tissues. pp. 45-117. In: Tumors in Domestic Animals, 4th ed. (Meuten, D. J. ed.), Iowa State Press, Ames, Iowa.

3. Kawanishi, S., Hiraku, Y., Pinlaor, S. and Ma, N. 2006. Oxidative and nitrative DNA damage in animals and patients with inflammatory diseases in relation to inflammation-related carcinogenesis. Biol. Chem. 387: 365-372.

4. Lala, P. K. and Chakraborty, C. 2001. Role of nitric oxide in carcinogenesis and tumour progression. Lancet Oncol. 2: 149156.

5. Moochhala, S. and Rajnakova, A. 1999. Role of nitric oxide in cancer biology. Free Rad. Res. 31: 671-679.

6. Ohshima, H., Tatemichi, M. and Sawa, T. 2003. Chemical basis of inflammation-induced carcinogenesis. Arch. Biochem. Biophys. 417: 3-11. 